\title{
La visión pragmatista de C.S. Peirce sobre la aserción
}

\author{
C.S. Peirce's pragmatist view of assertion
}

JAIME ALFARO IGLESIAS*

\begin{abstract}
Resumen: C.S. Peirce defendió una visión pragmatista de la aserción en términos de su efecto normativo. El presente artículo tiene dos objetivos. El primero es reconstruir y evaluar el argumento de Peirce a favor de la tesis de que aseverar una proposición es hacerse responsable de su verdad. El segundo es argumentar que Peirce interpretó "responsabilidad por la verdad" como la adquisición de un compromiso dialógico, a saber, el deber de defender la proposición aseverada dando razones ante desafío.

Palabras clave: pragmática de la aserción, pragmatismo, semántica dialógica.
\end{abstract}

\begin{abstract}
C.S. Peirce defended a pragmatist view of assertion in terms of its normative effect. This paper has two goals. First, to reconstruct and assess Peirce's argument for the thesis that to assert a proposition is to make oneself responsible for its truth. Second, to argue that Peirce interpreted "responsibility for truth" as the acquisition of a dialogical commitment, namely, the duty to defend the proposition asserted by giving reasons upon challenge.
\end{abstract}

Key words: pragmatics of assertion, pragmatism, dialogical semantics.

Aseverar es parte de nuestra vida cotidiana. Solemos aseverar mediante el uso de oraciones declarativas tales como "Está lloviendo" o "Dos más dos es igual a cuatro". Pero no toda enunciación de una oración declarativa es un acto de aserción. Al decir "Volveré mañana", por ejemplo, uno podría estar haciendo una promesa en vez de una aserción. Así, cabe preguntar: ¿qué hace alguien cuando asevera una proposición?' ${ }^{1}$ Un tipo de respuesta a esta pregunta es la llamada visión del "compromiso" de la aserción, la cual caracteriza los actos asertivos tales como afirmar, admitir, concluir, etc., en términos de su efecto normativo. ${ }^{2}$ El origen de esta visión, influyente en el debate contemporáneo sobre la aserción, ${ }^{3}$ se encuentra en la filosofía de C.S. Peirce, quien argumentó que "to assert a proposition is to make onself responsible for its truth" (CP 5.543). ${ }^{4}$

Fecha de recepción: 23/09/2015. Fecha de aceptación: 30/05/2017.

* Departamento de Filosofía, Universidade de São Paulo (Brasil). Investigador postdoctoral. Sus líneas de investigación son filosofía del lenguaje, filosofía e historia de la lógica y filosofía política. E-mail: alfaro@usp.br

1 Para una exposición panorámica del debate contemporáneo sobre la aserción, véase (Brown \& Cappelen, 2011).

2 Para una introducción a la teoría del compromiso de la aserción, véase (MacFarlane, 2011).

3 Robert Brandom, por ejemplo, sostiene que "to treat a performance as an assertion is to treat it as the undertaking or acknowledging of a certain kind of commitment [...]" (Brandom, 1994, 142). Por su parte, Searle sostiene que "asserting commits the speaker to the truth of the proposition asserted" (Searle, 2001, 147).Véase también (Wright, 1992).

4 Usaré las siguientes abreviaciones estándares para referirme a las ediciones de la obra de Peirce: CP (seguido del número de volumen y número de sección) = Collected Papers of Charles Sanders Peirce; EP (seguido del 
La visión de Peirce sobre la aserción y su argumento a favor de ésta son importantes por al menos dos razones. Por un lado, el debate contemporáneo sobre la aserción ha introducido visiones antagónicas a la visión del compromiso tales como la de que aseverar no tiene un efecto normativo, defendida por Peter Pagin (Pagin, 2004), y la de que no existe un acto de habla como la aserción, argumentada por Herman Cappelen (Cappelen, 2011). ${ }^{5}$ Por lo tanto, entender las razones de Peirce para defender la visión del compromiso puede contribuir a robustecer la situación dialéctica de dicho debate. Por otro lado, el concepto de aserción juega un rol central en la lógica deductiva de Peirce. Según Peirce, la hoja de aserción (sheet of assertion) representa el universo del discurso y las fórmulas que los lógicos inscriben en dicha hoja constituyen un signo que puede ser aseverado o, en términos de Peirce, una "proposición" (CP 4.430). ${ }^{6}$ Por lo tanto, esclarecer el significado de su visión sobre la aserción puede contribuir a una mejor comprensión de su lógica.

Sin embargo, la visión de Peirce sobre la aserción y sus razones en favor de ésta no han sido discutidas detenidamente por estudiosos de la filosofía de Peirce ni por filósofos del lenguaje. Así, el presente artículo tiene dos objetivos: (1) reconstruir y evaluar el argumento de Peirce para defender la visión del compromiso y (2) esclarecer la interpretación peirceana de la expresión "responsabilidad por la verdad".

Para la consecución de (1) y (2), procederé del siguiente modo. En la primera sección, me ocuparé del argumento de Peirce tal como éste es presentado en (CP 5.546). ${ }^{7}$ En particular, dada la exposición compendiosa de Peirce, explicaré algunas de las premisas y asunciones del argumento que requieren mayor elaboración. Finalmente, en la segunda sección, basado en la semántica dialógica para los cuantificadores existencial y universal expuesta por Peirce en (EP 2.168), argumentaré que Peirce interpretó "responsabilidad por la verdad" en términos dialógicos, es decir, como la adquisición del deber de defender la proposición aseverada dando razones ante desafío.

\section{El argumento de Peirce en (CP 5.546)}

Peirce defendió la tesis de que aseverar tiene un efecto normativo. Según Peirce, esta tesis significa que cuando uno asevera una proposición $p$, uno se hace responsable por la verdad de $p$. Según Peirce, el tipo de argumento adecuado para establecer la plausibilidad de esta tesis es el examen de un caso paradigmático de aserción. Peirce introdujo dicho argumento de la siguiente manera:

número de volumen y número de página) = The Essential Peirce; $\mathrm{W}$ (seguido del número de volumen y número de página) $=$ Writings of Charles $S$. Peirce: A Chronological Edition.

5 No hace parte del objetivo de este artículo presentar nuevas réplicas a estas visiones. Para una respuesta al argumento de Pagin, véase (Pegan, 2009). Para una respuesta a los argumentos de Cappelen, véase (Montgomery, 2014).

6 Asimismo, Peirce desarrolló una semántica para los cuantificadores existencial y universal en términos de diálogos asertivos (EP 2.168).

7 Si bien Peirce usa un acercamiento similar en (EP 2:140) y (EP 2:312), el manuscrito en el cual se basa el presente artículo presenta la exposición más clara y detallada sobre la naturaleza de la aserción ofrecida por Peirce en su filosofía madura. 
What is the nature of assertion? We have no magnifying-glass that can enlarge its features, and render them more discernible; but in default of such an instrument we can select for examination a very formal assertion, the features of which have purposely been rendered very prominent, in order to emphasize its solemnity. If a man desires to assert anything very solemnly, he takes such steps as will enable him to go before a magistrate or notary and take a binding oath to it. (CP 5.546)

Peirce pensó que las aserciones formales, tales como prestar juramento, son las candidatas naturales a aserciones paradigmáticas. A este respecto, Hilpinen menciona: "In his discussion of the nature of assertion Peirce adopted an interesting methodological view: to understand the nature of an assertion, we should study 'exaggerated' assertions in which the main features of the assertion are presented or displayed as clearly as possible." (Hilpinen, 1995, 273 $)^{8}$ Hilpinen destaca la visión metodológica de Peirce y la considera "[...] superior to the attempts to characterize concepts by necessary and sufficient conditions: the latter method has often prompted philosophers to be excessively concerned about 'difficult' and borderline cases, which has sometimes hampered fruitful philosophical theorizing." (Hilpinen, 1995, 274)

Si bien, como señala Hilpinen, el acercamiento de Peirce evita el excesivo énfasis en la discusión de casos límite, éste asume que los casos ordinarios se asemejan suficientemente a sus paradigmas. ¿Es plausible asumir que las aserciones ordinarias se asemejan suficientemente al acto de prestar juramento? Para responder a este interrogante, tomemos como punto de partida un ejemplo. Suponga que Adriana enuncia las siguientes oraciones en circunstancias adecuadas:

(a) Juro que Andrés robó mi bolso.

(b) Afirmo que Andrés robó mi bolso.

La presunción inicial de Peirce es que la enunciación de (a) y la de (b) constituyen diferentes formas de realizar el mismo acto de habla, esto es, aseverar la proposición de que Andrés robó el bolso de Adriana. Verbos tales como 'jurar' y 'afirmar' pertenecen a la categoría de los verbos asertivos. Algunos de los verbos en español que funcionan como verbos asertivos son los siguientes: afirmar, declarar, informar, predecir, admitir, concluir, asegurar, certificar, testimoniar y jurar. ${ }^{9}$ La categoría de los verbos asertivos reúne verbos mediante los cuales los hablantes confieren a lo que dicen un carácter factual. Sin embargo, estos verbos se diferencian entre sí en su fuerza asertiva. Por ejemplo, es claro que una aserción formal como (a) es más fuerte que una aserción ordinaria como (b). De hecho, verbos como 'jurar' y 'testimoniar' son asertivos fuertes mientras que verbos como 'afirmar' y 'sostener' son asertivos normales. Los verbos asertivos fuertes tienen una fuerza asertiva prominente y, como dijo Peirce, son usados cuando los hablantes desean enfatizar la solemnidad de un acto asertivo. De este modo, aunque los verbos asertivos se distinguen respecto a su fuerza,

8 Véase también (Hilpinen, 1982) y (Hilpinen, 1992).

9 Para una lista comprehensiva de verbos asertivos, véase (Searle \& Vandervaken, 1985). Véase (Hooper, 1975) para un tratamiento de los verbos asertivos desde un punto de vista lingüístico. 
todos estos son usados para hacer aserciones. ${ }^{10}$ Por lo tanto, es aceptable suponer que las aserciones ordinarias se asemejan suficientemente a sus paradigmas.

Retomo el argumento. Peirce pensó que las aserciones formales, las cuales están revestidas de un carácter solemne, son más adecuadas para examen que las aserciones ordinarias. Como mencioné anteriormente, dentro del conjunto de aserciones formales, Peirce seleccionó para examen un caso familiar, a saber, prestar juramento. Peirce continuó:

Taking an oath is not mainly an event of the nature of a setting forth, Vorstellung, or representing. It is not mere saying, but is doing. The law, I believe, calls it an "act." At any rate, it would be followed by very real effects, in case the substance of what is asserted should be proved untrue. This ingredient, the assuming of responsibility, which is so prominent in solemn assertion, must be present in every genuine assertion. (CP 5.546)

El punto de Peirce es claro: tomar un juramento no es simplemente decir sino hacer con lo que se dice. Suponga, por ejemplo, que María jura que Sócrates es barbado. Es claro que mediante este acto ella está representando a Sócrates de una determinada manera, a saber, teniendo pelo en las mejillas y la barbilla. En otras palabras, ella está diciendo algo acerca de Sócrates. Por lo tanto, si uno jura una proposición, entonces uno dice algo. Sin embargo, tomar juramento no consiste meramente en representar personas u objetos de cierta manera. Quien jura una proposición se convierte en un agente en virtud de ejecutar dicho acto y, dado que todo agente es responsable por las consecuencias de sus actos, quien toma juramento es responsable por lo que dice. Por ejemplo, suponga que Adriana testifica bajo juramento que Andrés le robó el bolso y, como resultado, Andrés es detenido. Suponga ahora que Andrés no robó el bolso de Adriana. ¿Cuáles son los "efectos reales" de que la proposición aseverada por Adriana sea falsa? Evidentemente, el juez habría tomado la decisión errada de detener a Andrés. Por lo tanto, Adriana habría cometido perjurio y sería legal y moralmente punible. Tal como lo ilustra el ejemplo, es claro que el estatus normativo de Adriana cambió en virtud de haber proferido un juramento $y$, consecuentemente, ella adquirió una responsabilidad manifiesta respecto a la proposición que juró, a saber, se hizo responsable de su verdad.

El punto de Peirce es que los hablantes que aseveran son agentes y, en esa condición, son responsables por sus actos dentro de la comunidad lingüística. A este respecto Karl Otto Apel comentó:

Ceremonious statements that explicitly take responsibility for their content, such as an oath before a court, merely make visible, as through a magnifying glass, the willful, morally relevant, active aspect that is inherent in every assertion [...]. Not just the oath as a legal act, but every assertion that implicitly claims truth is an action by which the one making the assertion enters into reality in a causal, dynamic way and becomes engaged morally in the communication community. (Apel, 1981, 163)

10 Por supuesto, es posible reconocer verbos asertivos débiles tales como 'conjeturar', 'especular', etc., cuya fuerza asertiva es menor que la de los verbos asertivos normales. 
Según Apel, Peirce pensó que los hablantes se convierten en agentes morales cuando participan del discurso asertórico. Por lo tanto, las aserciones de los hablantes pueden ser evaluadas como correctas o incorrectas y, de este modo, los hablantes qua agentes deben asumir una responsabilidad moral por sus afirmaciones. Así, según Peirce, aseverar no es mero decir, pues este acto coloca al hablante en un escenario moral dentro de la comunidad comunicativa. Sin duda, estas consideraciones otorgan suficiente plausibilidad a la premisa de que si un hablante jura una proposición, entonces el hablante es un agente moral.

En suma, Peirce infirió que quienes prestan juramento son responsables a partir de la premisa de que quienes juran son agentes morales. Dado que los hablantes juran proposiciones y las proposiciones son falsas o verdaderas, es claro que cuando un hablante jura una proposición, éste se hace responsable de la verdad de dicha proposición. Y todo agente moral es punible mediante crítica por los efectos negativos de sus acciones. Por lo tanto, quien asevera es punible si asevera una proposición falsa. Examinemos con mayor detenimiento este punto.

Peirce afirmó que el acto de tomar juramento tiene efectos reales en caso de que la proposición aseverada sea falsa. ¿Qué significa esta afirmación? ¿A qué clase de "efectos reales" se refiere Peirce? La inspección de Peirce de prestar juramento muestra que mediante la aserción formal que $p$, quien asevera respalda $p$ e insta al oyente a asentir a $p$. Por "asentir", Peirce entendió "[...] an act of the mind by which one endeavors to impress the meaning of the proposition upon his [the addressee's] disposition, so that it shall govern his conduct, including thought under conduct [...]" (EP 2.278). Esto significa que si uno asiente a una proposición, uno está dispuesto a usarla en las circunstancias adecuadas. En otras palabras, al realizar una aserción formal, quien asevera aprueba la proposición aseverada y, de este modo, recomienda abiertamente al oyente a creer esta proposición. Por lo tanto, es claro que quien jura que $p$ avala $p$ ante su interlocutor. Esto significa que el primero da al último derecho a re-aseverar que $p$, aseverar cualquiera de sus consecuencias, y, por lo tanto, tomar $p$ como base para el razonamiento práctico y la acción. ${ }^{11}$

El punto de Peirce es que si el interlocutor tiene derecho a usar la proposición aseverada por su contraparte para efectos de su razonamiento práctico y acción, entonces quien asevera es punible mediante crítica moral por los daños o efectos negativos que podrían ser evitados en caso de que la proposición aseverada sea falsa. Peirce escribió:

But if a lie would not endanger the esteem in which the utterer was held, nor otherwise be apt to entail such real effects as he could avoid, the interpreter would have no reason to believe the assertion. Nobody takes any positive stock in those conventional utterances, such as "I am perfectly delighted to see you," upon whose falsehood no punishment at all is visited. (CP 5.546)

11 Debo señalar que Peirce no introdujo explícitamente la distinción entre los compromisos directos e indirectos. Suponga que Elena afirma que la rosa es roja. Se sigue de lo que Elena afirmó que alguna rosa es colorida. Decimos entonces que Elena está directamente comprometida con la proposición de que la rosa es roja e indirectamente comprometida con la proposición de que alguna rosa es colorida. En general, se dice que cuando uno afirma que $p$, uno está indirectamente comprometido con las consecuencias de $p$. Para esta distinción, véase (Brandom, 1994). 
Por mor del argumento, suponga que quienes aseveran no son moralmente punibles por "los efectos reales" que podría causar a sus interlocutores creer una proposición falsa. Según Peirce, si esto fuera el caso, se seguiría que los interlocutores son indiferentes acerca de los efectos desafortunados que se pueden derivar de creer una proposición falsa. Por ejemplo, los jueces serían indiferentes si enviasen a alguien a la cárcel con base en falsos testimonios, el común de las personas sería indiferente si recibiese direcciones equivocadas, los matemáticos serían indiferentes si sus colegas usasen conjeturas a manera de premisas en las pruebas, etc. Queda claro que la conclusión de que los interlocutores son indiferentes respecto a creer una proposición falsa es inaceptable por sus efectos cognitivos y prácticos. Por lo tanto, quienes aseveran son punibles mediante crítica moral si las proposiciones que aseveran son falsas (a menos que tengan una excusa adecuada). En suma, quienes aseveran son susceptibles de crítica moral si aseveran una proposición falsa debido al daño que podría causar a sus destinatarios creer tal proposición.

No obstante, cabe señalar que existen casos en que un hablante asevera una proposición falsa y no es moralmente punible. La razón de esto es que un hablante puede ofrecer una excusa suficiente para haber aseverado incorrectamente. Una comparación con el acto de prometer puede ofrecer luz sobre este punto. ${ }^{12}$ Suponga, por ejemplo, que Elena se compromete a cuidar del gato de Julia mientras ésta se va de vacaciones. Sin embargo, Elena olvida alimentar al gato y finalmente el gato muere de inanición. Es claro que Elena es punible mediante crítica moral simpliciter. Suponga ahora que Elena olvida alimentarlo debido a una amnesia transitoria ocasionada por un golpe en su cabeza. La situación ha cambiado drásticamente, pues ahora Elena tiene una excusa válida. Así, se dice que Elena es responsable, al menos retrospectivamente, pero no punible. Asimismo, ser responsable por la verdad de una proposición no implica punibilidad: es posible que quien ha aseverado una proposición falsa tenga una excusa adecuada para haberlo hecho. Al incluir esta salvedad, la visión de Peirce sobre la aserción involucra al acto de retractación, esto es, el acto de revocar lo dicho, el cual es consistente con nuestras prácticas comunicativas ordinarias.

De este modo, Peirce concluyó que la adquisición de la responsabilidad por parte de quien asevera, el cambio en su estatus normativo, es el efecto esencial de tomar juramento. Dado que las aserciones ordinarias se asemejan suficientemente a sus paradigmas, se sigue que la tesis de que aseverar tiene un efecto normativo es plausible. Presento a continuación una reconstrucción sucinta del argumento de Peirce:

(i) Las aserciones ordinarias se asemejan suficientemente a sus paradigmas.

(ii) Si un hablante $h$ jura una proposición $p$, entonces $h$ es un agente.

(iii) Si $h$ es un agente en virtud de jurar $p$, entonces $h$ es responsable por la verdad de $p$ y punible mediante crítica si $p$ resulta ser falsa (a menos que $h$ tenga una excusa suficiente).

Por lo tanto,

12 Para un análisis comparativo de los actos de aseverar y prometer, véase (Watson, 2004). 
(C) Aseverar tiene un efecto normativo: Si $h$ asevera que $p$, $h$ es responsable de la verdad de $p$.

Podría objetarse que el tipo de argumento propuesto por Peirce no puede proporcionar una prueba concluyente de que aseverar tiene un efecto normativo. Una respuesta a esta objeción es que el argumento de Peirce no pretende ofrecer tal prueba. El propósito del argumento de Peirce es establecer la plausibilidad de la tesis de que aseverar tiene un efecto normativo. Por lo tanto, la imposibilidad de proporcionar una prueba para esta tesis no constituye un problema.

Finalmente, es pertinente mencionar que, aunque el argumento reconstruido establece la conclusión de que aseverar tiene un efecto normativo, este argumento no establece directamente la afirmación de que la aserción debe ser caracterizada en términos de dicho efecto. ${ }^{13}$ Esto no debe sorprendernos, pues Peirce adoptó el principio del pragmatismo como un principio metodológico tácito. Tal principio dice que existe una conexión entre el contenido de un concepto y sus efectos prácticos (EP:346). Así, siguiendo el principio del pragmatismo, la visión de Peirce sobre la aserción puede ser formulada de la siguiente manera:

(A)Un hablante $h$ asevera una proposición $p$ si y sólo si $h$ se hace responsable por la verdad de $p$.

Sin embargo, dado que los hablantes no tienen ninguna influencia en la verdad de las proposiciones, el significado de la parte derecha de (A) requiere mayor elaboración: ¿en qué sentido un hablante es "responsable por la verdad" de una proposición? En la siguiente sección me ocuparé de este interrogante.

\section{2. ¿Qué significa "responsabilidad por la verdad"?}

Tomaré como punto de partida para esclarecer el significado de la expresión "responsabilidad por la verdad" la visión de Peirce sobre el concepto de verdad. Peirce no intentó responder las preguntas clásicas “¿Qué es la verdad?” o “¿Cómo establecer que una determinada creencia es verdadera?” Por contraste, Peirce se ocupó de la siguiente pregunta: ¿qué hacemos al tomar una proposición como verdadera? Considérese el siguiente fragmento:

[...] You only puzzle yourself by talking of this metaphysical "truth" and metaphysical "falsity," that you know nothing about. [...] if by truth and falsity you mean something not definable in terms of doubt and belief in any way, then you are talking of entities of whose existence you can know nothing, and which Ockham's razor would clean shave off. Your problems would be greatly simplified, if, instead of saying that you

13 Tal como (Chauiviré, 1979) sugiere, la visión de Peirce sobre la aserción es un buen ejemplo de cómo él usó su célebre principio del pragmatismo. Este principio dice que uno debe analizar los conceptos en términos de sus efectos prácticos concebibles (EP 1:132; EP 2:346). Sin embargo, debo recalcar que Peirce no fue explícito en su uso del principio del pragmatismo para efectos de establecer su visión de la aserción. Para un estudio de las diversas formulaciones de este principio y su lugar en la filosofía de Peirce, véase (Hookway, 2012). 
want to know the "Truth," you were simply to say that you want to attain a state of belief unassailable by doubt. (EP 2.336)

Aquí Peirce rechaza todas las teorías de la verdad con arraigo metafísico, por ejemplo, versiones de la teoría de la correspondencia, pues estas teorías presuponen una perspectiva sub specie aeternitatis para ser inteligibles. Esta presuposición significa que la verdad es una relación, entre nuestras afirmaciones y la realidad, totalmente independiente de nuestra vida cognitiva. Según Peirce, el problema con dicha presuposición es que de ella se sigue la imposibilidad de confrontar nuestras afirmaciones con una realidad a la que no tenemos acceso.

Peirce propone entender la noción de verdad a partir de la interacción dialéctica entre creer y dudar. Peirce caracterizó "creer" como un estado de estabilidad en el cual uno está dispuesto a usar sus creencias casi irreflexivamente en el razonamiento y la acción. Evidentemente, si uno encuentra evidencia recalcitrante contra una creencia, la duda surge impetuosamente. Así, Peirce caracterizó dudar como un estado de perturbación e irritación, un estado en el que el razonamiento y la acción ya no se acoplan a la creencia (W 3: 246). Como resultado, si uno duda, entonces uno desea poner fin a la perturbación mediante la fijación de una creencia. En otras palabras, uno está dispuesto a actuar para alcanzar un estado imperturbable por la duda. Según Peirce, la actividad más adecuada para alcanzar dicho estado es la investigación (inquiry). Peirce interpretó "investigación” como una actividad social, pues es llevada a cabo por la comunidad de investigadores; y normativa, pues tiene por finalidad evaluar la corrección de nuestros estados doxásticos estableciendo de forma crítica si las proposiciones que creemos son indubitables. Para Peirce, dicho aspecto crítico, el cual consiste en el embate entre razones para creer y razones para dudar, es el que confiere idoneidad a la investigación como método de fijación de creencias. Consecuentemente, según Peirce, una proposición verdadera es aquella que es indudable al final de la investigación (EP 2.204; 336, 347). ${ }^{14}$

La visión de Peirce sobre la verdad sirve de trasfondo para entender la noción de responsabilidad por la verdad presentada por Peirce al final de (CP 5.546): “[...] every assertion involves an effort to make the intended interpreter believe what is asserted, to which end a reason for believing it must be furnished" (CP 5.546). ${ }^{15}$ Peirce pensó que, en una comunidad de hablantes, hacerse responsable de la verdad de una proposición es comprometerse a hacer algo, a saber, defender dicha proposición ofreciendo razones. A la luz de este pasaje, podemos reformular (A) del siguiente modo:

$\left(\mathrm{A}^{1}\right)$ Un hablante $h$ asevera una proposición $p$ si y sólo si $h$ se compromete a defender $p$ dando razones.

Considérense dos casos para ilustrar $\left(\mathrm{A}^{1}\right)$. En el primer caso, María asevera que el gato está en el sofá. Si Orlando le pregunta: “Cómo lo sabes?”, ella puede honrar su responsabilidad por la verdad de la proposición aseverada respondiendo "Acabo de ver al gato" o

14 Para un estudio de la visión de Peirce sobre la noción de verdad, véase (Misak, 1991).

15 A la luz de este pasaje, la conexión entre aseverar e investigar radica en que ambas son prácticas socio-normativas de dar y pedir razones propias de la comunidad de hablantes y de investigadores. 
“¡Ven aquí y mira!”. En el segundo caso, un matemático afirma que la ecuación $x^{n}+y^{n}=$ $z^{n}$ no tiene solución en los números enteros cuando $n$ es mayor que 2, a menos que $x y z=$ 0. ¿Qué debe hacer el matemático para honrar su responsabilidad por la verdad? Presentar una prueba de dicha proposición.

Sin embargo, la anterior interpretación de "responsabilidad por la verdad" presenta una dificultad. Si aceptamos que (A) es equivalente a $\left(\mathrm{A}^{1}\right)$, entonces tendremos que aceptar que Peirce desdibujó la diferencia entre los conceptos de verdad y justificación. Siguiendo a Putnam (1981: 55), debemos caer en la cuenta de que una proposición puede estar justificada en determinado momento y a la postre resultar falsa. Por ejemplo, hace tres mil años alguien podía comprometerse a dar razones en favor de la proposición de que la tierra es plana a partir de los estándares científicos de la época. Sin embargo, no consideramos que dicha proposición era verdadera hace tres mil años y hoy es falsa. Mientras justificar una proposición es algo relativo a diversos factores tales como la disponibilidad de evidencias y lo que en cada contexto cuente como evidencia admisible, la verdad de una proposición no es relativa a esos factores. Por lo tanto, "es justificado" y "es verdadero" no son expresiones sinónimas. De este modo, asumiendo que Peirce no confundió los conceptos de verdad y justificación, cabe preguntar: ¿propone la visión de Peirce sobre la aserción un compromiso con la verdad objetiva de la proposición aseverada o con la capacidad de justificarla adecuadamente?

Para responder esta pregunta es necesario considerar los aspectos social y normativo de la aserción. No cabe duda de que Peirce sostuvo que una proposición es verdadera o falsa y que no tenemos ninguna injerencia sobre la verdad o falsedad de una proposición (EP 2.224). Sin embargo, Peirce ofreció una importante salvedad respecto a este punto de vista: una proposición no puede per se proporcionar razones para considerarla verdadera o falsa (EP 2.276). En otras palabras, Peirce consideró que no debemos diseccionar la noción de verdad y, luego, escoger entre el aspecto objetivo y el cognitivo de la verdad. Si bien no debemos renunciar a pensar que una proposición verdadera lo es de manera objetiva, tampoco debemos renunciar a pensar que necesitamos razones para considerarla verdadera.

No es sorprendente, a la luz de las anteriores consideraciones, que Peirce intentara sintetizar los aspectos objetivo y cognitivo del concepto de verdad en su visión sobre la aserción. Y dicha síntesis tiene lugar en la noción de evaluación. Ahora, antes de abordar esta noción, es pertinente distinguir dos sentidos de evaluación respecto al acto de aseverar.

En primer lugar, mediante una evaluación normativa, podemos preguntar si un acto de aserción es correcto en el sentido de si el hablante tiene permitido hacerlo en virtud de tener razones para defender la proposición. Por ejemplo, considérese de nuevo a María aseverando que el gato está en el sofá. María asevera correctamente si está en capacidad de dar razones en favor de lo que dice, es decir, si está en capacidad de mostrar el gato en el sofá. No obstante, pese a que su acto es normativamente correcto, es posible que la proposición aseverada por María sea falsa en virtud de la información de que dispone en el momento de aseverar o, simplemente, porque María está equivocada.

En segundo lugar, mediante una evaluación objetiva, podemos preguntar si un acto de aserción es correcto en el sentido de si la proposición aseverada es verdadera, es decir, en el sentido de si las cosas son tal como se dice que son. Permítaseme detallar esta noción 
considerando la dirección de ajuste (direction of fit) del acto de aseverar una proposición. ${ }^{16}$ El acto de aseverar tiene dirección de ajuste de palabra a mundo, porque la aserción correcta de una proposición debe coincidir con el mundo. Dado que los hablantes aseveran proposiciones, que pueden ser verdaderas o falsas, el valor de verdad de una proposición debe originarse en la frontera donde el lenguaje se encuentra con el mundo. Para Peirce esa frontera es la acción:

Every proposition refers to some index: universal propositions to the universe, through the environment common to speaker and auditor, which is an index of what the speaker is talking about. But the particular proposition asserts that, with sufficient means, in that universe would be found an object to which the subject term would be applicable, and to which further examination would prove that the image called up by the predicate was also applicable. (CP 2.369)

Como sugiere este pasaje, la evaluación objetiva no consiste en asignar valores de verdad a las proposiciones sino en señalar el modo correcto de aplicar expresiones a los miembros de un conjunto de cosas y no a los de otro. Peirce pensó que un hablante asevera correctamente si es posible mostrar que el uso del predicado aplicado al objeto (u objetos) de la proposición en cuestión se ajusta al mundo. Por ejemplo, volviendo al caso de María, el acto de aserción sería objetivamente correcto si fuese posible comprobar que María aplicó correctamente el predicado "está en el sofá" a Ferio, el gato de casa, en el momento de su aserción.

La evaluación normativa y la evaluación objetiva son inteligibles para Peirce en un escenario dialógico. Dichos sentidos de evaluación son correlativos al contraste de perspectivas entre la primera y la segunda persona. La perspectiva de la segunda persona es aquella que $T U ́$ y $Y O$ adoptamos cuando evaluamos la corrección de un acto, en este caso un acto de aserción. ${ }^{17}$ Estas perspectivas no presuponen una meta-perspectiva que acceda, como el "ojo de Dios", a una realidad en sí. Las perspectivas de la primera y la segunda persona cooperan (antagónicamente) para evaluar la corrección objetiva normativa, por así decirlo, del acto de aserción. ${ }^{18}$ Este punto, tal como mostraré a continuación, es soportado y ejemplificado por la semántica para los cuantificadores universal y existencial propuesta por Peirce en términos de diálogos asertivos.

Peirce interpretó el cuantificador universal y el cuantificador existencial de la siguiente manera: "Some' means that the speaker is to select an instance, while 'Every' or 'Any' means that a second person is to perform the selection" (CP 2.523). ${ }^{19}$ Así, la semántica dialógica de Peirce para los cuantificadores puede ser introducida informalmente a seguir: $P$ simboliza el proponente en tanto defensor y $O$ simboliza el oponente en tanto atacante.

16 Como (Searle, 1979), quien a su vez sigue a (Anscombe, 1963), entiendo por "dirección de ajuste" la dirección de acople entre el mundo y un acto de habla de aserción que se requiere para que este último sea correcto.

17 Para un estudio de la perspectiva de la segunda persona, véase (Darwall, 2006).

18 (Brandom, 1994) emplea la noción de evaluación objetiva normativa. No obstante, tal como (Habermas, 2000) ha argumentado, la visión de Brandom presenta problemas ya que sustituye el rol de la segunda persona por el de la tercera persona.

19 (Marion, 2012) ofrece una breve introducción a la semántica dialógica y presenta a Peirce como uno de los pioneros de este acercamiento. 
El diálogo comienza con $P$ aseverando una oración cuantificada y $O$ desafiándola, con los interlocutores alternando turnos. Las reglas que capturan el significado de los cuantificadores son las siguientes: cuando $P$ asevera $\forall x F(x), O$ selecciona un valor para $x$ y entonces $P$ debe mostrar que el predicado $F$ aplica al valor seleccionado para $x$. Cuando $P$ asevera $\exists x F(x), O$ solicita a $P$ que elija un valor para $x$ para el cual el predicado $F$ aplique. Aclararé el importe de estas reglas y su relación con la noción de responsabilidad por la verdad mediante el siguiente ejemplo ofrecido por Peirce:

[...] If I guarantee to find a miserable sinner, of course, I guarantee there is a sinner in the world. But if I turn the responsibility of picking out the sinner to you, I do not guarantee you can find one. I only say if you do find one, he will turn out miserable. This is the distinction between Universal and Particular propositions. (CP 2.453)

Nótese que Peirce usa las expresiones "if I guarantee" y "if I turn the responsibility of picking up the sinner to you", las cuales ejemplifican cómo el hablante y su interlocutor alternan roles en aras de evaluar la aserción de una proposición cuantificada. Adaptaré el ejemplo de Peirce a un contexto específico. Suponga que un sacerdote y una feligresa conversan tranquilamente. El sacerdote asevera que algún pecador es infeliz. El diálogo para esta aserción inicia con la feligresa ejerciendo su derecho a desafiar. El sacerdote debe defender la proposición aseverada mediante la selección de un pecador que sea infeliz. Ahora, suponga que el sacerdote asevera que todos los pecadores son infelices. Por contraste con la primera aserción, la feligresa tiene el derecho de seleccionar un pecador arbitrario. La feligresa encuentra uno muy feliz, ella misma. El sacerdote no logra mostrar que la feligresa es infeliz. Dado que existe al menos un pecador feliz, el sacerdote se retracta de su aserción.

Según los diálogos asertivos para las proposiciones cuantificadas, el hablante que asevera y su interlocutor alternan roles en aras de evaluar si el hablante aseveró correctamente. Por lo tanto, es claro que Peirce construyó la relación entre hablante e interlocutor como una relación simétrica. Asimismo, dado que Peirce concibió el interlocutor como un oponente, quien tiene el derecho a desafiar, se sigue que Peirce construyó la perspectiva del interlocutor como una perspectiva de segunda persona. A la luz de estas consideraciones, podemos reformular $\left(\mathrm{A}^{1}\right)$ del siguiente modo:

$\left(\mathrm{A}^{2}\right)$ Un hablante $h$ asevera una proposición $p$ si y sólo si $h$ se compromete a defender $p$ dando razones ante un interlocutor $i$ que tiene el derecho a atacar $p$ pidiendo razones.

En $\left(\mathrm{A}^{2}\right)$, la noción de responsabilidad por la verdad es resignificada a partir de la diferencia de perspectivas entre la primera y la segunda persona dando lugar a la noción de compromiso dialógico: el hablante que asevera, el proponente, adopta el compromiso de dar razones y su interlocutor, el oponente, atribuye al proponente dicho compromiso y, por ende, tiene el derecho de pedir razones.

¿Por qué el hablante que asevera adopta un compromiso dialógico? Desde la perspectiva de un hablante en primera persona, las proposiciones justificadas son equivalentes a las proposiciones verdaderas. Quien asevera correctamente, desde la perspectiva de la primera persona, debe honrar el canon normativo de evaluación, es decir, se compromete a dar razo- 
nes. Por contraste, desde la perspectiva de un interlocutor en segunda persona, del supuesto de que YO (el proponente, quien asevera) esté en capacidad de defender las proposiciones aseveradas dando razones, no se sigue necesariamente que estas proposiciones sean verdaderas. Es aquí donde el rol de la segunda persona cobra importancia. Siguiendo a Peirce en (CP 2.369), en el diálogo entre oponente y proponente, el embate entre perspectivas permite honrar el canon objetivo de evaluación y determinar si el predicado de la oración aplica al objeto referido por el nombre. Por lo tanto, las perspectivas de la primera y la segunda persona cooperan para evaluar la corrección objetivo-normativa de un acto de aserción. Esta es la interpretación dialógica de Peirce de "responsabilidad por la verdad".

\section{Conclusión}

En la primera parte de este artículo, reconstruí y evalué el argumento de Peirce a favor de la tesis de que el efecto de los actos asertivos consiste en el cambio del estatus normativo del hablante. Basado en esta conclusión, Peirce propuso una visión pragmatista de la aserción. En la segunda parte, me ocupé de esclarecer que un hablante es "responsable por la verdad" de una proposición en el sentido de comprometerse a defender dicha proposición dando razones. Sin embargo, este sentido mostró ser problemático dada la distinción entre los conceptos de verdad y justificación. Así, apelé a la noción de evaluación de un acto de aserción, la cual tiene un aspecto normativo y un aspecto objetivo. Basado en la semántica dialógica de Peirce para los cuantificadores, argumenté que Peirce consideró que la práctica de la aserción está estructurada de manera que el hablante qua proponente y su interlocutor qua oponente puedan cooperar con el objetivo de determinar si la aserción del hablante es correcta o incorrecta. En este marco, la noción de evaluación "objetiva normativa" cobra sentido a partir del diálogo y la oposición simétrica entre las perspectivas de la primera y la segunda persona.

No cabe duda de que especificar todas las implicaciones de la visión de Peirce sobre la aserción, así como posibles objeciones a éstas, es un proyecto mucho más complejo. En el presente artículo sólo he intentado reconstruir y evaluar las razones que Peirce ofreció a favor de la visión del compromiso de la aserción y esclarecer su interpretación de ésta. Según dicha evaluación e interpretación, Peirce dio buenas razones para creer que su visión ofrece una imagen convincente de la aserción a partir de la simple idea de que los actos asertivos tienen un efecto normativo característico, a saber, la adquisición de un compromiso dialógico entre hablante e interlocutor. ${ }^{20}$

\section{Bibliografía}

Anscombe, E. (1963), Intention, 2nd Ed., Oxford: Basil Blackwell.

Apel, K.O. (1981), Charles S. Peirce: from pragmatism to pragmaticism, Amherst, MA: University of Massachusetts Press.

20 Agradezco a los evaluadores anónimos por sus comentarios y sugerencias a la versión anterior de este artículo. Asimismo, agradezco a Adriana Madriñán Molina por sus aportes y sugerencias idiomáticas. 
Brandom, R. (1994), Making it explicit: Reasoning, Representing, and Discursive Commitment. Cambridge, MA: Harvard University Press.

Brown, J., \& Cappelen, H. Eds. (2011), Assertion: New Philosophical Essays, Oxford: Oxford University Press.

Cappelen, H. (2011), “Against assertion”, in Brown, J. \& Cappelen, H. (eds.) Assertion: New Philosophical Essays, Oxford: Oxford University Press.

Chauviré, C. (1979), "Peirce, le langage et l'action, sur la théorie peircienne de l'assertion", Les Études philosophiques 1: 3-17.

Darwall, S. (2006). The Second-Person Standpoint: Morality, Respect, and Accountability, Cambridge, MA: Harvard University Press

Goldberg, S. (2015), Assertion: On the Philosophical Significance of Assertoric Speech, Oxford: Oxford University Press.

Habermas, J. 2000. "From Kant to Hegel: On Robert Brandom's Pragmatic Philosophy of Language", European Journal of Philosophy, 8 (3):322-55.

Hilpinen, R. (1982), “On C. S. Peirce's theory of the proposition: Peirce as a precursor of game-theoretical semantics", The Monist 65: 182-188.

Hilpinen, R. (1992), “On Peirce's Philosophical Logic: Propositions and Their Objects", Transactions of the Charles S. Peirce Society 28 (3): 467-488.

Hilpinen, R. (1995), "Peirce on Language and Reference", in Laine, K., (ed.) Peirce and Contemporary Thought: Philosophical Inquiries. Fordham: Fordham University Press.

Hookway, C. (2012), The Pragmatic Maxim, Oxford: Oxford University Press.

Hooper, J. (1975), "On assertive predicates", in Syntax and Semantics, ed. John P. Kimball, 91-124. New York: Academic Press.

MacFarlane, J. (2011), "What is assertion?", in Brown, J. \& Cappelen, H. (eds.) Assertion: New Philosophical Essays, Oxford: Oxford University Press.

Marion, M. (2012), "Game semantics and the manifestation thesis" in Rahman, S., Primero, G., \& Marion, M., Eds., The Realism-Antirealism Debate in the Age of Alternative Logics. Logic, Epistemology, and the Unity of Science Volume 23. Berlin: Springer, pp. 141-168.

Misak, C.J. (1991), Truth and the End of Inquiry: A Peircian Account of Truth, Oxford: Clarendon Press.

Montgomery, B. (2014), "In defense of assertion”, Philosophical Studies, 171 (2): 313-326.

Pagin, P. (2004), “Is assertion social? “, Journal of Pragmatics, 36 (5): 833-59.

Pegan, P. (2009), "Why assertion may yet be social”, Journal of Pragmatics, 41(12): $2557-$ 2562.

Peirce, C. S. (1931-1935), Collected Papers of Charles Sanders Peirce, voll. 1-6. Edited by Hartshorne, C. \& Weiss, P. Cambridge, MA: Harvard University Press;

Peirce, C. S. (1958), Collected Papers of Charles Sanders Peirce, voll. 7-8. Edited by Burks, A. Cambridge, MA: Harvard University Press.

Peirce, C. S. (1998), The Essential Peirce. Edited by Kloesel, C. \& Houser, N., vol. 2. Bloomington: Indiana University Press.

Peirce, C. S. (1982-2000), Writings of Charles S. Peirce: A Chronological Edition. Ed. by Peirce Edition Project. Bloomington: Indiana University Press.

Putnam, H. (1981), Reason, Truth and History, Cambridge: Cambridge University Press. 
Searle, J. (1979), Expression and Meaning: Studies in the Theory of Speech Acts, Cambridge: Cambridge University Press.

Searle, J. (2001), Rationality in Action, Cambridge: MIT Press.

Searle, J. and Vanderveken, D. (1985), Foundations of Illocutionary Logic, Cambridge: Cambridge University Press.

Watson, G. (2004), “Asserting and promising”. Philosophical Studies, 117 (1-2):57-77.

Wright, C. (1992), Truth and Objectivity. Cambridge, MA: Harvard University Press. 